Claremont Colleges

Scholarship@ Claremont

All HMC Faculty Publications and Research

HMC Faculty Scholarship

$12-27-2001$

\title{
Scroll Waves in the Presence of Slowly Varying Anisotropy with Application to the Heart
}

S. Setayeshgar

California Institute of Technology

Andrew J. Bernoff

Harvey Mudd College

\section{Recommended Citation}

S. Setayeshgar \& A. J. Bernoff, "Scroll Waves in the Presence of Slowly Varying Anisotropy with Applications to the Heart", Phys. Rev. Lett. 88 (2002).

This Article is brought to you for free and open access by the HMC Faculty Scholarship at Scholarship @ Claremont. It has been accepted for inclusion in All HMC Faculty Publications and Research by an authorized administrator of Scholarship @ Claremont. For more information, please contact scholarship@cuc.claremont.edu. 


\title{
Scroll Waves in the Presence of Slowly Varying Anisotropy with Application to the Heart
}

\author{
S. Setayeshgar* \\ Department of Applied Mathematics, California Institute of Technology, Pasadena, California 91125
}

Andrew J. Bernoff

Department of Mathematics, Harvey Mudd College, Claremont, California 91711

(Received 11 June 2001; published 27 December 2001)

\begin{abstract}
We consider the dynamics of scroll waves in the presence of rotating anisotropy, a model of the left ventricle of the heart in which the orientation of fibers in successive layers of tissue rotates. By choosing a coordinate system aligned with the fiber rotation and studying the phase dynamics of a straight but twisted scroll wave, we derive a Burgers' equation with forcing associated with the fiber rotation rate. We present asymptotic solutions for scroll twist, verified by numerics, using a realistic fiber distribution profile. We make connection with earlier numerical and analytical work on scroll dynamics.
\end{abstract}

DOI: 10.1103/PhysRevLett.88.028101

A central question in the field of cardiac dynamics is the decay mechanism of ventricular tachycardia to fibrillation [1]. Tachycardia is thought to be characterized by scroll waves of electrical wave activity, with an intrinsic frequency (120 to 500 beats $/ \mathrm{min}$ ) more than twice that of the heart's natural pacemaker. Fibrillation is spatiotemporally incoherent electrical wave activity (greater than 500 beats/min), leading to failure of the heart's pumping, which rapidly results in death. Growing experimental evidence indicates that spiral waves (or scroll waves in three dimensions) of electrical activity in cardiac tissue are related to fatal arrhythmias [2]. It has been widely conjectured that the instability of a single spiral to a spatiotemporally disordered state governs the transition from tachychardia to fibrillation.

The focus of the present work is to explore the effect of the strong anisotropy present in cardiac tissue on the dynamics of scroll waves. Panfilov and Keener [3] showed numerically that rotating anisotropy could lead to scroll wave breakup in three dimensions. Fenton and Karma [4] have related this instability to the formation of localized regions of large twist. Previous analytical studies [5,6] have addressed the dynamics of scroll waves in isotropic excitable media. In this Letter, we extend these works, demonstrating analytically for the first time the destabilizing role fiber rotation may have on scroll wave dynamics.

Dissection results indicate that the left ventricle consists of nested layers of cardiac muscle fibers [7]. Streeter et al. [8] presented photomicrographs of successive layers of ventricular tissue showing alignment of the elongated
PACS numbers: $87.10 .+\mathrm{e}, 02.30 . \mathrm{Mv}$, 87.18.Pj

muscle fibers in each layer, and gradual rotation of their orientation between layers as one proceeds transmurally from the outermost (epicardium) to the innermost (endocardium) layer. As electrical conductivity along the fibers is several times faster than in the perpendicular direction, the fiber architecture of the ventricle gives rise to a rotating anisotropy, shown schematically in Fig. 1. The $z$ axis corresponds to the transmural direction, and $\Theta(z)$ is the angle between the fast axis of diffusion and the $x$ axis.

The ratios of the fast/slow diffusion coefficients inplane $\left(D_{\|} / D_{\perp_{1}}\right)$ and out-of-plane $\left(D_{\|} / D_{\perp_{2}}\right)$ are approximately equal for cardiac tissue and have been measured to be between 4 and 9 [9]. Peskin [10] proposed a fiber distribution, $\Theta(z)$, to be

$$
\Theta(z)=\sin ^{-1}(z / r L)
$$

where $-L<z<L$, and $r \geq 1$ is a cutoff parameter, determining the total fiber rotation through the thickness of the ventricle. This agrees well with measured results [8].

The rotating anisotropy suggests a natural coordinate system in which the new coordinate axes rotate with the fiber orientation, $\Theta(z)$, and are rescaled by the in-plane anisotropy, $\alpha=\sqrt{D_{\|} / D_{\perp_{1}}}$ :

$$
\left(\begin{array}{c}
x \\
y / \alpha \\
z
\end{array}\right)=\left(\begin{array}{ccc}
\cos \Theta(\tilde{z}) & \sin \Theta(\tilde{z}) & 0 \\
-\sin \Theta(\tilde{z}) & \cos \Theta(\tilde{z}) & 0 \\
0 & 0 & 1
\end{array}\right)\left(\begin{array}{c}
\tilde{x} \\
\tilde{y} \\
\tilde{z}
\end{array}\right),
$$

where $(\tilde{x}, \tilde{y}, \tilde{z})$ denote the fixed Cartesian coordinates (cf. Fig. 1).

The governing equations in the new coordinates are

$$
\begin{aligned}
\vec{u}_{t}=\vec{f}(\vec{u})+\boldsymbol{D}_{\|} & \cdot \Delta_{2} \vec{u}+\boldsymbol{D}_{\perp_{2}} \cdot \frac{\partial^{2} \vec{u}}{\partial z^{2}} \\
+\boldsymbol{D}_{\perp_{2}} \cdot & \left\{\Theta^{\prime 2}\left[\frac{\partial^{2}}{\partial \theta^{2}}+\left(\alpha^{2}-1\right) x^{2} \frac{\partial^{2}}{\partial y^{2}}+\left(\frac{1}{\alpha^{2}}-1\right) y^{2} \frac{\partial^{2}}{\partial x^{2}}\right] \vec{u}\right. \\
& \left.\quad 2 \Theta^{\prime}\left[\frac{\partial}{\partial \theta}+(\alpha-1) x \frac{\partial}{\partial y}-\left(\frac{1}{\alpha}-1\right) y \frac{\partial}{\partial x}\right] \frac{\partial \vec{u}}{\partial z}-\Theta^{\prime \prime}\left[\frac{\partial}{\partial \theta}+(\alpha-1) x \frac{\partial}{\partial y}-\left(\frac{1}{\alpha}-1\right) y \frac{\partial}{\partial x}\right] \vec{u}\right\},
\end{aligned}
$$



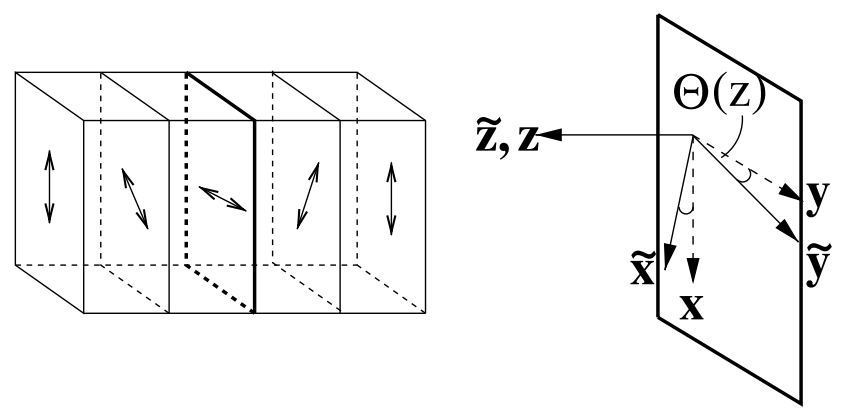

FIG. 1. Schematic diagram of the rotating anisotropy of cardiac tissue: The $z$ axis denotes the direction through the thickness of the tissue. The coordinates $(\tilde{x}, \tilde{y}, \tilde{z})$ are fixed in the lab, while $(x, y, z)$ rotate with the fiber direction. Here the fibers are aligned with the $y$ axis.

where $\vec{f}$ denotes the nonlinear reaction kinetics, $\vec{u}$ is a vector of concentrations (for example, the transmembrane potential and gating variables in the case of a cardiac tissue), $\Delta_{2}$ is the two-dimensional Laplacian, and $\Theta^{\prime}(z)$ denotes the fiber rotation rate (FRR). In the absence of fiber rotation $\left[\Theta^{\prime}(z)=0\right]$ a simple rescaling by $\sqrt{D_{\|} / D_{\perp_{2}}}$ retrieves the isotropic governing equation and solution. In the following, all terms involving both the anisotropy and the fiber rotation rate will be treated as a perturbation.

Working in the new coordinate system allows us to identify a dimensionless small parameter $\epsilon$ for the perturbation analysis. To do so, we assume a solution given by $\vec{u}(\vec{r}, t)=\vec{U}_{0}\left[r, \theta-\omega_{0} t+\Theta(z)+\phi(z, t)\right]+\epsilon^{2} \vec{u}_{2}(\vec{r}, t)$,

where the base solution $\vec{U}_{0}\left[r, \theta-\omega_{0} t+\Theta(z)\right]$ is the straight, untwisted scroll in the original coordinates for $\alpha=1$, and $\omega_{0}$ is its frequency. Here we treat the simplest case of a straight filament. [We relax this assumption, accounting for the motion of the filament and its coupling to the phase dynamics in [11].] The validity of this assumption is based on previous work showing numerically the existence of a finite threshold of twist below which a twisted, straight scroll remains straight in an isotropic excitable medium [12]. Note that for the filament to be straight in both coordinate systems, it is necessary to choose the $z$ axis to lie along the scroll filament. [Wellner et al. [13] have treated the intramural scroll wave (whose filament lies within a fiber plane).]

The base solution is perturbed by a phase $\phi(z, t)$ which is assumed to vary slowly in space and time:

$$
\phi_{t} / \omega_{0} \sim \mathcal{O}\left(\epsilon^{2}\right), \quad \ell \phi_{z} \sim \mathcal{O}(\epsilon),
$$

where $\ell$ is a transverse length scale determined below. Substituting this solution into the governing equation, de-dimensionalizing according to $\hat{t}=\omega_{0} t, \hat{z}=z / \ell$, and dropping hats, the linear equation for $\vec{u}_{2}$ at $\mathcal{O}\left(\epsilon^{2}\right)$ is

$$
\epsilon^{2} \mathcal{L} \vec{u}_{2}=\vec{g},
$$

where $\mathcal{L} \equiv \omega_{0} \partial_{t}-\boldsymbol{D}_{\|} \Delta_{2}-\boldsymbol{F}\left(\vec{U}_{0}\right)$ is a linear differential operator and $\boldsymbol{F}=\left.\vec{f}_{\vec{u}}\right|_{\vec{U}_{0}}$, with

$$
\begin{aligned}
\vec{g}=-\omega_{0}\left(\frac{\partial \phi}{\partial t}\right) \vec{U}_{0_{\theta}}+\left(\boldsymbol{D}_{\perp_{2}} / \ell^{2}\right) \cdot & \left\{\left(\frac{\partial \phi}{\partial z}\right)^{2} \vec{U}_{0_{\theta \theta}}+\left(\frac{\partial^{2} \phi}{\partial z^{2}}\right) \vec{U}_{0_{\theta}}+h_{1}^{2}(z)\left[\mathcal{H}_{1} \vec{U}_{0}-2 \mathcal{H}_{2} \vec{U}_{0_{\theta}}\right]\right. \\
& \left.-2 h_{1}(z)\left(\frac{\partial \phi}{\partial z}\right) \mathcal{H}_{2} \vec{U}_{0_{\theta}}-h_{2}(z) \mathcal{H}_{2} \vec{U}_{0}\right\} .
\end{aligned}
$$

The operators $\mathcal{H}_{1}$ and $\mathcal{H}_{2}$ are

$$
\begin{aligned}
& \mathcal{H}_{1}=\left(\frac{1}{\alpha^{2}}-1\right) y^{2} \partial_{x}^{2}+\left(\alpha^{2}-1\right) x^{2} \partial_{y}^{2}, \\
& \mathcal{H}_{2}=(\alpha-1) x \partial_{y}-\left(\frac{1}{\alpha}-1\right) y \partial_{x},
\end{aligned}
$$

and

$$
h_{1}(z)=\frac{\ell}{r L} \frac{1}{\sqrt{1-(\ell z / r L)^{2}}}, \quad h_{2}(z)=h_{1}^{\prime}(z) .
$$

The transverse length scale $\ell$ is determined by balancing the first three terms in $\vec{g}$, giving $\ell^{2}=D_{\perp_{2}} / \omega_{0}$. Requiring all terms in $\vec{g}$ to be $\leq \mathcal{O}\left(\epsilon^{2}\right)$, the physical small parameter, $\epsilon$, is

$$
\epsilon^{2}=\frac{D_{\perp_{2}}}{\omega_{0} L^{2}} \frac{\gamma^{2} / 4-1}{r^{2}-1},
$$

where $\gamma(\alpha)=\alpha+1 / \alpha$. Three dimensionless constants contribute to $\epsilon$ : (i) $\left(D_{\perp_{2}} / \omega_{0} L^{2}\right)$, the ratio of the transverse diffusion length to the system size, (ii) $1 /\left(r^{2}-1\right)$, determined by the (maximum) fiber rotation rate, and (iii) $\left(\gamma^{2} / 4-1\right)$, which measures the anisotropy and van- ishes in the isotropic case. It is this product that must be "small."

Estimates for $\epsilon$ vary between species and depend on the thickness of the left ventricular wall, $2 L$. Consistent with other works we take $r=1.5$, corresponding to a total fiber angle of $120^{\circ}$. For the human heart, the relevant parameters are $2 L=1 \mathrm{~cm}, D_{\|}=1 \mathrm{~cm}^{2} \mathrm{~s}^{-1}, D_{\perp}=0.1 \mathrm{~cm}^{2} \mathrm{~s}^{-1}$, and $\nu=2 \mathrm{~s}^{-1}$ [14]. With these parameters, we find $\epsilon^{2} \sim 0.45$.

A solvability condition yields the phase equation at $\mathcal{O}\left(\epsilon^{2}\right)$ :

$$
\begin{array}{r}
\frac{\partial \phi}{\partial t}-c_{1}\left(\frac{\partial \phi}{\partial z}\right)^{2}-c_{2} \frac{\partial^{2} \phi}{\partial z^{2}}=c_{3} h_{1}^{2}(z)-c_{5} h_{2}(z) \\
-2 c_{4} h_{1}(z) \frac{\partial \phi}{\partial z},
\end{array}
$$

where $c_{3}=\left(\gamma^{2}-4\right) a_{1}-(\gamma-2) c_{1}, c_{4}=\left(\frac{1}{2} \gamma-1\right) c_{1}$, and $c_{5}=\left(\frac{1}{2} \gamma-1\right) c_{2}$. The coefficients $\left(a_{1}, c_{1}, c_{2}\right)$ are given by

$$
\begin{gathered}
a_{1}=\left\langle\vec{Y}_{0}, \boldsymbol{D}_{\perp_{2}} \cdot x^{2} \vec{U}_{0_{y y}}\right\rangle=\left\langle\vec{Y}_{0}, \boldsymbol{D}_{\perp_{2}} \cdot y^{2} \vec{U}_{0_{x x}}\right\rangle, \\
c_{1}=\left\langle\vec{Y}_{0}, \boldsymbol{D}_{\perp_{2}} \cdot \vec{U}_{0_{\theta \theta}}\right\rangle, \quad c_{2}=\left\langle\vec{Y}_{0}, \boldsymbol{D}_{\perp_{2}} \cdot \vec{U}_{0_{\theta}}\right\rangle .
\end{gathered}
$$


In the preceding equation, the inner product $\langle$,$\rangle is$

$$
\left\langle\overrightarrow{\boldsymbol{v}}_{1}, \overrightarrow{\boldsymbol{v}}_{2}\right\rangle=\int_{0}^{2 \pi / \omega_{o}} d t \int_{0}^{2 \pi} d \theta \int_{0}^{\infty} r d r \overrightarrow{\boldsymbol{v}}_{1} \cdot \overrightarrow{\boldsymbol{v}}_{2} .
$$

Here $\vec{Y}_{0}$ is a null vector of the adjoint linear operator, $\mathcal{L}^{\dagger} \equiv \partial_{t}-\boldsymbol{D}_{\|} \Delta_{2}-\boldsymbol{F}^{\top}\left(\vec{U}_{0}\right)$, such that $\left\langle\vec{Y}_{0}, \vec{U}_{0_{\theta}}\right\rangle=1$, where $\vec{U}_{0_{\theta}}$ is the corresponding null vector of the linear operator $\mathcal{L}$ (assumed to be a Fredholm operator of index zero). The details of the perturbation analysis follow closely the treatments by others $[5,15]$, and are given elsewhere [11]. There is no coupling to translational modes since the filament is assumed to be straight and stationary.

Introducing a new variable,

$$
\Phi(z, t) \equiv\left(\frac{c_{1}}{c_{2}}\right)\left[\phi(z, t)-\left(\frac{\gamma}{2}-1\right) \Theta(z)\right],
$$

and rescaling $t \rightarrow t\left(c_{2} \ell^{2} / L^{2}\right)$ and $z \rightarrow z \ell / L$ reduces the phase equation to the forced Burgers' equation:

$$
\Phi_{t}-\Phi_{z}^{2}-\Phi_{z z}=A(r) F(z ; r),
$$

where the forcing function is given by

$$
F(z ; r)=\frac{1-1 / r^{2}}{1-(z / r)^{2}},
$$

and

$$
A(r)=\tilde{A} \frac{\gamma^{2} / 4-1}{r^{2}-1}, \quad \tilde{A}=\left(\frac{c_{1}}{c_{2}}\right)^{2}\left(\frac{4 a_{1}}{c_{1}}-1\right),
$$

for $-1<z<1$. Determining $\tilde{A}$ requires either numerical computation of the null space of $\mathcal{L}^{\dagger}$ or simulation of the full three-dimensional governing equations, allowing for extraction of $\left(a_{1}, c_{1}, c_{2}\right)$ from the phase dynamics. Both are numerically challenging, and have not yet been undertaken; hence, these values are not explicitly known. We do know that the "diffusion" constant, $c_{2}$, is positive and equal to $D_{\perp_{2}}$ for equally diffusive species. As diffusion would be destabilizing for negative $c_{2}$, we expect it to be positive for the singly diffusive FitzHugh-Nagumo kinetics, based on the stability of 3D numerical solutions [11].

With no anisotropy, the forcing term is identically zero $(A=0)$, and the known, unforced Burgers' equation is retrieved [5]. If FRR is constant, the unforced Burgers' equation can again be obtained, by introducing a constant frequency shift. However, the right-hand side is nontrivial for nonconstant FRR.

The physiologically relevant boundary conditions are no-flux at the vertical boundaries (epi-/endocardium). This condition is exact if the FRR vanishes on the boundary or in the absence of anisotropy. Otherwise, it holds in an averaged sense as the scroll rotates if $\Phi_{z}=0$. We now examine the behavior of the solution to Eq. (5) in certain limits with these boundary conditions. We look for solutions with a constant frequency shift, $\lambda$ :

$$
\Phi(z, t)=\int_{-1}^{z} k\left(z^{\prime}\right) d z^{\prime}+\lambda t+\Phi_{0},
$$

where $\Phi_{0}$ is a constant. Furthermore, given the asymmetric fiber distribution profile, the solution $\Phi_{z}(z, t)$ is also odd under $z \rightarrow-z$. Hence, we look for solutions with $k( \pm 1)=k(0)=0$.

a. Diffusive regime $\left(\Phi_{z z} \gg \Phi_{z}^{2}\right)$. - This solution is formally valid when either $|A| \ll 1$ or $|r| \gg 1$, and is given by

$$
k(z)=A(r)\left(1-\frac{1}{r^{2}}\right)\left[z \tanh ^{-1}\left(\frac{1}{r}\right)-\tanh ^{-1}\left(\frac{z}{r}\right)\right],
$$

where

$$
\lambda=A(r)\left(1-\frac{1}{r^{2}}\right) \tanh ^{-1}\left(\frac{1}{r}\right) .
$$

This numerical solution to Eq. (5) is shown in Fig. 2. In this limit, the phase equation is linear, and the sign of the forcing is irrelevant.

b. Twist-dominated regime $\left(\Phi_{z z} \ll \Phi_{z}^{2}\right)$. - This solution is formally valid when $|A| \gg 1$. In this case, we must distinguish between the two signs of forcing. It is useful to transform the forced Burgers' equation via the ColeHopf transformation, $k(z)=\psi_{z}(z) / \psi(z)$, to the timeindependent Schrödinger equation:

$$
\frac{d^{2} \psi}{d z^{2}}+[-\lambda-V(z)] \psi=0,
$$

where $V(z)=\mp A(r) F(z ; r)$, with $(\mp)$ corresponding to $( \pm)$ forcing. The ground state is given by the smallest $|\lambda|$ and the corresponding eigenfunction satisfying the symmetry and boundary conditions. This ground state "energy" is set by the behavior of the potential in the vicinity of its minimum on $-1<z<1$, which occurs at the boundary for positive forcing and at the origin for negative forcing.

Maintaining the first two terms in the expansion of the potential, with $\lambda=\lambda_{0}+\lambda_{1}$, gives the following results.

(i) Negative forcing: The Schrödinger equation for the one-dimensional harmonic oscillator is obtained, with

$$
\lambda_{0}=-\bar{A} / r^{2}, \quad \lambda_{1}=-\bar{A}^{1 / 2} / r^{2},
$$

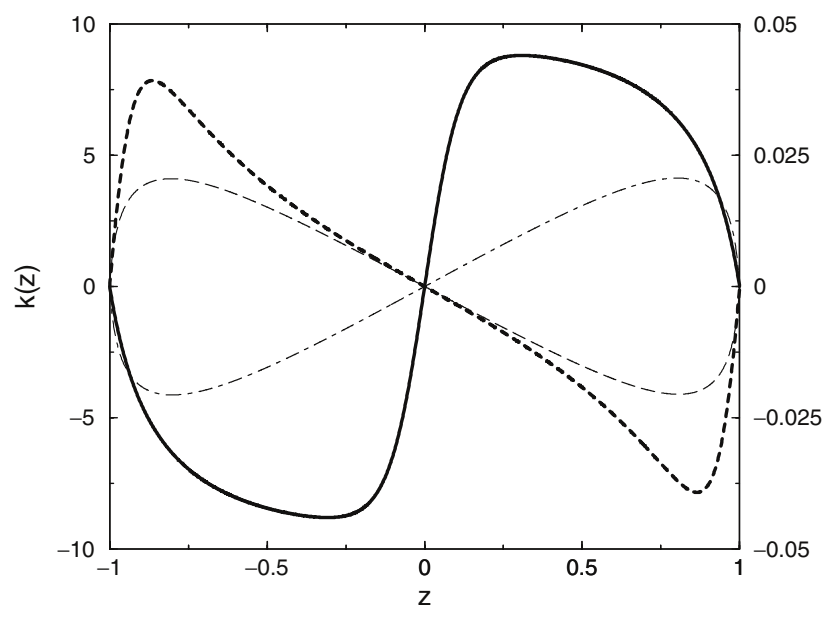

FIG. 2. Left axis: large forcing, $|A(r)|=227.3$ and $r=1.2$. The solid line corresponds to $A(r)>0$, and the dashed line to $A(r)<0$. Right axis: small forcing, $|A(r)|=1.0$ and $r=$ 1.01. The dot-dashed line corresponds to $A(r)>0$, and the long-dashed line corresponds to $A(r)<0$. 
where $\bar{A}=\tilde{A}\left(\frac{\gamma^{2}}{4}-1\right)$. In Fig. 2, the steady state solution $k(z)$ for large negative forcing (dashed line) indicates expulsion of twist from the bulk to boundary layers at the boundaries.

(ii) Positive forcing: The Airy equation is obtained, with

$$
\lambda_{0}=\bar{A} /\left(r^{2}-1\right), \quad \lambda_{1}=\eta(2 \bar{A})^{2 / 3} /\left(r^{2}-1\right)^{4 / 3},
$$

where $\eta$ is the first zero of $A i^{\prime}(z)$. For this case, Fig. 2 (solid line) shows the formation of large twist in a boundary layer in the bulk.

The analytical results for the frequency $\lambda$ with large negative and positive forcing have been verified with numerical evolution of the forced Burgers' equation and are in good agreement over a large range of $|A| \gg 1$.

Twist is given by the variation of the phase of the scroll along the scroll filament. For a straight scroll, with the filament given by the $z$ axis, it is the spatial derivative of the phase in the new coordinates:

$$
w(z, t)=\phi_{z}(z, t)+\Theta^{\prime}(z) .
$$

There exists a large body of numerical and analytical work on scroll twist, mostly in isotropic excitable media [12,16,17]. Recently, Margerit and Barkley [18] have performed an asymptotic analysis of uniformly twisted straight scrolls in the low excitability limit, deriving the shape and frequency of waves at leading and first order. As Winfree has pointed out, twist occurs naturally in physical excitable systems: for example, in cardiac tissue, a transmural gradient in the electrophysiological properties of the medium, resulting in the scroll wave rotating at different frequencies on the inner and outer surfaces, leads to a twisted scroll. Our phase equation extends these works to describe the dynamics of twist in the presence of rotating anisotropy.

By applying the coordinate transformation (1), twist in the old coordinates is

$$
w(\tilde{z}, t)=\Theta^{\prime}(\tilde{z})-\frac{2 \alpha\left[\phi_{\tilde{z}}(\tilde{z}, t)+\Theta^{\prime}(\tilde{z})\right]}{\left(\alpha^{2}-1\right) \cos 2\left[\omega_{0} t-\phi(\tilde{z}, t)-\Theta(\tilde{z})\right]+\left(\alpha^{2}+1\right)} .
$$

We note a time dependence at twice the frequency of the unperturbed simple scroll. Although this subharmonic behavior has received attention [4], it is clear from our formulation of twist in the new coordinate system that this is an artifact of rotating anisotropic diffusion. Rather, it is the phase dynamics $\phi(z, t)$ that is relevant in the possible formation of a twist singularity.

In summary, by identifying the natural coordinate system to be given by the rotation of the muscle fiber orientation in cardiac tissue, we have derived a forced Burgers equation for the evolution of scroll twist. We found numerical and asymptotic solutions in the limits of small and large forcing using Peskin's derived fiber distribution profile. For small forcing, the maximum value of twist occurs below the boundaries. For large forcing, the sign of forcing determines whether this maximum occurs in the bulk or is expelled to the boundaries. Given the numerical evidence [11,12] for the buckling instability of a scroll filament above a finite threshold of twist, our results suggest a combined role of electrophysiology, fiber architecture, and anisotropy, reflected in the forcing constants $\tilde{A}$, $r$, and $\gamma$, respectively, in such an instability. These results demonstrate that rotating anisotropy generates scroll twist, pointing to the possible destabilizing role of cardiac tissue structure on scroll phase dynamics (for example, through the "sproing" instability). Our finding reinforces the need for taking this realistic feature into account in model studies.

A. J.B. acknowledges support from the NSF DMS9971969. S. S. thanks Herb Keller and Alain Karma for useful discussions, and acknowledges support from the American Heart Association while at Northeastern University (Grant No. 96009660) and the NSF (CCR-9120008) while at the Center for Research on Parallel Computation at Caltech.

\section{*Corresponding author.}

Email address: simas@math.princeton.edu

[1] For a collection of articles, see Chaos (1998).

[2] W. F. Witkowski et al., Nature (London) 392, 78 (1998).

[3] A. V. Panfilov and J. P. Keener, Physica (Amsterdam) 84D, 545 (1995).

[4] F. Fenton and A. Karma, Chaos 8, 20 (1998).

[5] J. P. Keener, Physica (Amsterdam) 31D, 269 (1988); J. P. Keener and J. J. Tyson, Physica (Amsterdam) 44D, 191 (1990); 53D, 151 (1991); SIAM Rev. 34, 1 (1992).

[6] V. N. Biktashev, Physica (Amsterdam) 36D, 167 (1989); 40D, 83 (1989).

[7] C. E. Thomas, Am. J. Anat. 101, 17 (1957).

[8] D. D. Streeter, D. P. Patel, J. Ross, and E. H. Sonnenblick, Circ. Res. 24, 339 (1969); D. D. Streeter, W. E. Powers, M. A. Ross, and F. Torrent-Guasp, in Cardiovascular System Dynamics, edited by J. Baan, A. Noordergraaf, and J. Raines (M.I.T. Press, Cambridge, 1978), p. 73.

[9] A. T. Winfree, Int. J. Bifurcation Chaos Appl. Sci. Eng. 7, 487 (1997).

[10] C. S. Peskin, Commun. Pure Appl. Math. 42, 79 (1989).

[11] S. Setayeshgar and A. J. Bernoff (to be published).

[12] C. Henzi, E. Lugosi, and A. T. Winfree, Can. J. Phys. 68, 683 (1990).

[13] M. Wellner, O. Berenfeld, and A. M. Pertsov, Phys. Rev. E 61, 1845 (2000).

[14] A. T. Winfree, in Progress in Biophysics and Molecular Biology, edited by D. Noble et al. (Pergamon Press, Oxford, New York, 1997).

[15] V. N. Biktashev, A. V. Holden, and H. Zhang, Philos. Trans. R. Soc. London A 347, 611 (1994).

[16] A. T. Winfree, SIAM Rev. 32, 1 (1990).

[17] A. S. Mikhailov, A. V. Panfilov, and A. N. Rudenko, Phys. Lett. A 109, 246 (1985); A. V. Panfilov and A. T. Winfree, Physica (Amsterdam) 17D, 323 (1985).

[18] D. Margerit and D. Barkley, Phys. Rev. Lett. 86, 175 (2001). 